\title{
Editorial for the Special Issue on 3D Data Processing, Visualization and Transmission
}

\author{
Adrien Bartoli • Marcus Magnor • Bob Fisher • \\ Christian Theobalt
}

Received: 1 December 2011 / Accepted: 2 December 2011 / Published online: 10 December 2011

(C) Springer Science+Business Media, LLC 2011

This special issue discusses new results, technologies and applications related to the capture, representation, processing, storage, transmission and visualization of $3 \mathrm{D}$ geometric and photometric models.

The guest editors selected 7 papers from 128 as candidates for this publication. The topics of the accepted papers cover several important problems in the area of 3D vision. The first four papers discuss image registration through keypoint detection and matching. "Physical Scale Keypoints: Matching and Registration for Combined Intensity/Range Images" by Smith et al. presents a method to detect and match keypoints between images for which the depth of each pixel is known. "Interesting Interest Points-A Comparative Study of Interest Point Performance on a Unique Data Set" by Aanæs et al. presents a framework for the evaluation of keypoint detection with ground truth, using the recall rate as main criterion. The conference version of this paper received the Best Paper Award at 3DPVT 2010. "Imposing Semi-Local Geometric Constraints for Accurate Correspondences Selection in Structure from Motion: A GameTheoretic Perspective" by Albarelli et al. discusses the prob-

A. Bartoli $(\bowtie)$

ISIT, Université d'Auvergne, Clermont-Ferrand, France

e-mail: adrien.bartoli@gmail.com

M. Magnor

TU Braunschweig, Braunschweig, Germany

e-mail: m.magnor@tu-bs.de

B. Fisher

University of Edinburgh, Edinburgh, UK

e-mail: rbf@inf.ed.ac.uk

C. Theobalt

MPI Informatik, Saarbruecken, Germany

e-mail: theobalt@mpii.de lem of keypoint matching using semi-local constraints computable from local image features. The conference version of this paper received the NVidia Best Student Paper Award at 3DPVT 2010. "Feature-Based Deformable Surface Detection with Self-Occlusion Reasoning" by Pizarro et al. uses local geometric models to constrain keypoint matching for images of deformable surfaces. The next two papers deal with 3D shape inference from images. "On Camera Calibration with Linear Programming and Loop Constraint Linearization" by Courchay et al. proposes a Structure-fromMotion approach that combines partial reconstructions represented by matching tensors while taking multiple overlaps into accounts. "Self-calibrated, Multi-spectral Photometric Stereo for 3D Face Capture" by Vogiatzis et al. proposes a multi-spectral 3D reconstruction system for faces with an integrated calibration procedure using Structure-from-Motion. Finally, "Automatic Real-Time Video Matting Using Timeof-Flight Camera and Multichannel Poisson Equations" by Wang et al. combines color and depth to compute alpha matting.

We believe that this particular set of exciting papers illustrates how well research in the area of 3D imaging is progressing. We thank the authors and the reviewers for their contributions to this special issue.

\section{Guest Editors}

